\title{
Perceived Barriers to Mammography Adoption among Women in Iran: A Qualitative Study
}

\author{
Shayesteh Shirzadi', Hamid Allahverdipour ${ }^{2, *}$, Manoj Sharma ${ }^{3}$, Hadi Hasankhani ${ }^{4}$ \\ 'Department of Health Education and Promotion, Tabriz University of Medical Sciences, Tabriz, Iran \\ ${ }^{2}$ Research Center of Psychiatry and Behavioral Sciences, Department of Health Education and Promotion, Tabriz University of Medical Sciences, \\ Tabriz, Iran \\ ${ }^{3}$ Department of Behavioral \& Environmental Health, School of Public Health, Jackson State University, Jackson, MS, USA \\ ${ }^{4}$ Department of Nursing, Qualitative Research Center, Tabriz University of Medical Sciences, Tabriz, Iran
}

Background: Breast cancer is the most commonly diagnosed tumorous cancer and the second leading cause of cancer-related deaths among women worldwide. This study aimed to qualitatively explore the barriers to mammography adoption among Iranian women.

Methods: This study is a qualitative component of a large research project on exploratory sequential mixed method design, utilizing conventional content analysis. In total, 24 participants were selected from among women who had participated in the first phase of the study. Data were collected through in-depth, semi-structured interviews.

Results: Five main themes were extracted, consisting of unawareness of mammography, fear control, priority of mammography needs, inadequate competency of mammography centers, and a sense of losing family support. Conclusion: Different perceived barriers within various levels (individual, intrapersonal, health systems, and community) play influential roles in women's decisions to participate in breast cancer screening program, which indicates the cultural aspect of perceived barriers in different communities and countries. The study provides the basis for intervention planning in this regard.

Keywords: Qualitative Study; Breast Neoplasms; Mammography; Self-Appraisal

Received: April 13, 2018, Revised: July 6, 2018, Accepted: July 19, 2018

*Corresponding Author: Hamid Allahverdipour https://orcid.org/0000-0003-3700-6185

Tel: +98-411-3344731, Fax: +98-411-3344731, E-mail: allahverdipourh@tbzmed.ac.ir 


\section{INTRODUCTION}

Breast cancer is the most commonly diagnosed tumorous cancer and the second leading cause of cancer-related deaths worldwide. ${ }^{1)} \mathrm{Be}-$ sides that, the treatment costs for breast cancer are high and impose a huge economic burden on countries because of high rates of incidence and prevalence. ${ }^{2)}$ Although breast cancer incidence and prevalence are increasing, breast cancer mortality in high-income countries is decreasing because of early detection through mammogram screening and therapeutic advances at the community level. ${ }^{3)}$ Because the stage of cancer is considered to be the most important factor for the prognosis of most types of cancers and breast cancer is the leading reason for reduction of cancer-related mortality, early detection greatly contributes to decreasing the burden of cancer. ${ }^{4)}$ Women's awareness plays an important role in the effective diagnosis of breast cancer at an early stage. ${ }^{5)}$ As a result, early diagnosis can help to improve survival, select less-invasive treatments, and achieve a higher quality of life, thus reducing the disease burden and treatment costs. Increasing incidence of breast cancer in low- and middle-income countries has led to numerous challenges for the healthcare providers, health authorities, and civil societies of these countries, resulting in placing the need for greater emphasis on the early detection of breast cancer. ${ }^{6}$

Previous studies have identified various barriers to breast cancer screening behaviors. These barriers include difficulties in accessing screening services; the cultural context of the community; personal concerns regarding screening procedure; concerns regarding the sensitivity and efficacy of mammogram screening; pain $;{ }^{7,8)}$ psychosocial issues, such as fear of being diagnosed with breast cancer; ${ }^{7)}$ cost; ${ }^{9)}$ low perceived susceptibility; ${ }^{10)}$ and fear of malignant breast tumor detection. ${ }^{11)}$ Several studies on the early detection of breast cancer in Asian women have focused on sociodemographic characteristics, whereas cultural factors are not very well understood and may also have important relationships with breast cancer screening behaviors. ${ }^{12)}$

Certain types of barriers may be more or less important for specific cultural or ethnic subpopulations. In this regard, modesty has been reported as a specific barrier to adherence to mammography screening among Asian American women. Fear, embarrassment, and cost are the most common barriers to adherence among African American women. ${ }^{13)}$ Besides that, among Arab women who are living in the United States, in addition to the abovementioned barriers, lack of adopting health screening practices, stigmatization of cancer, fear, and ignorance about breast cancer screening were also found to be the barriers to mammography. Furthermore, certain culture-specific barriers have also been reported, including embarrassment, family relationships, fatalism, and consultation with unprofessional and elderly women. ${ }^{14)}$

Thus, further efforts should be made to motivate women to undergo screening and diagnostic mammogram procedures. ${ }^{3)}$ Given these data, effective factors on early detection of breast cancer should be explored; therefore, this study aimed to explore culture-specific barriers to mammography among Iranian women.

\section{METHODS}

The present study was the qualitative part of a large research project om exploratory sequential mixed method design performed with the aim of explaining the barriers to mammography among Iranian women to develop a scale of mammography barriers. To achieve this purpose, a qualitative study design using conventional content analysis was adopted. Twenty-four women were selected from among those who had participated in the first phase of our study. The participants consisted of 17 women without history of mammography, six with history of mammography or family history of breast cancer, and one mammography technician (Table 1). Ethical approval for the study protocol was provided by the Ethics Committee of Tabriz University of Medical Sciences (ethics code: IR.TBZMED.REC. A/149-451). All the participants voluntarily signed the informed consent form to participate in the study before the interviews.

\section{Data Collection}

Data were collected through individual, in-depth, and semi-structured interviews. Each interview began with open-ended question: "Why do you not participate in mammography?" First, women without a history of mammography were interviewed, and after achieving data saturation, other women were interviewed to supplement the data. The subsequent questions asked were based on the participants' responses. The participants were also allowed to select the time and location of the interviews. Each participant was interviewed in a session lasting approximately 20-60 minutes. All interviews were recorded using a voice recorder.

\section{Data Analysis}

Data were analyzed using conventional qualitative content analysis. For this purpose, all interviews were transcribed verbatim and then carefully read several times. The analysis started by identifying units of

Table 1. Sociodemographic characteristics of participants

\begin{tabular}{lc}
\hline \multicolumn{1}{c}{ Characteristic } & No. (\%) \\
\hline Had no history of mammography & $17(70.8)$ \\
Had history of mammography or family history of breast cancer & $6(25)$ \\
Mammography technician & $1(4.2)$ \\
Age (y) & \\
$40-50$ & $15(62.5)$ \\
$\geq 50$ & $9(37.5)$ \\
Educational status & \\
Elementary and secondary level & $6(25)$ \\
High school graduate & $4(16.7)$ \\
University level & $14(58.3)$ \\
Marital status & \\
$\quad$ Married & $19(79.2)$ \\
Single/divorced/widowed & $5(20.8)$ \\
Occupation & \\
Does not work outside home & $4(16.7)$ \\
Employed outside home & $20(83.3)$ \\
Total & $24(100)$ \\
\hline
\end{tabular}


meaning that were drawn from the transcripts. Codes were generated inductively and were assigned to different categories based on differences and similarities. Data analysis continued until data saturation was achieved so that no other themes could be drawn.

\section{Data Trustworthiness}

To supplement the trustworthiness of data, a good relationship was established between the interviewers and participants and adequate time was allocated for data collection. In addition, the interview transcripts and codes drawn were presented to the participants to elicit their viewpoints regarding the units of meaning. In addition to the research team, the transcripts were also presented to a number of experts, as external observers, who were not involved in the study, so that they could examine the accuracy of the coding process.

\section{RESULTS}

In total, five categories and 12 subcategories were extracted. The categories consisted of unawareness of mammography, fear control, prioritizing the need for mammography, inadequate competency of mammography centers, and a sense of losing family support (Table 2).

\section{Unawareness of Mammography}

This category comprised of two subcategories, (1) lack of information among women about breast cancer and mammograms and (2) misunderstandings.

\section{1) Lack of information among women about breast cancer and mammograms}

The participants' information about mammography and breast cancer was inadequate or incomplete. They believed that breast cancer was an asymptomatic disease and is diagnosed only in the progressive stages after the onset of pain. The participants did not also have adequate information about the consequences of late diagnosis of breast cancer and importance of its early detection. Moreover, some women were not familiar with the terms 'mammography' and 'screening' and their applications. Most of the women believed that mammogram was

Table 2. Classification of main categories and subcategories

\begin{tabular}{|c|c|}
\hline Main categories & Subcategories \\
\hline Unawareness on mammography & $\begin{array}{l}\text { Lack of information for mammogram } \\
\text { Misunderstanding }\end{array}$ \\
\hline Fear control & $\begin{array}{l}\text { Perceived fear } \\
\text { Denying risk of breast cancer } \\
\text { Breast conflict }\end{array}$ \\
\hline $\begin{array}{l}\text { Need prioritization vs. } \\
\text { mammography preference }\end{array}$ & $\begin{array}{l}\text { Prioritize needs } \\
\text { Difficulties in referring to mammography centers } \\
\text { Lack of income/economic barriers }\end{array}$ \\
\hline $\begin{array}{l}\text { Inadequate competency of } \\
\text { mammography centers }\end{array}$ & $\begin{array}{l}\text { Lack of mammography centers and experts } \\
\text { Clients-health professional relationship }\end{array}$ \\
\hline $\begin{array}{l}\text { A sense of losing familial } \\
\text { support }\end{array}$ & $\begin{array}{l}\text { Loss of family support } \\
\text { Disrupted body image stigma }\end{array}$ \\
\hline
\end{tabular}

not necessary unless certain changes in the breast such as lumps and pain occurred and received suspicious ultrasound or clinical examination results. The participants also complained of poor training regarding health-related subjects such as mammography, screening, and breast cancer. They expressed that women and girls were poorly informed regarding the early detection of breast cancer, leading to lack of women's referring to healthcare centers. In addition, most women believed that they only need to undergo mammography as per the healthcare provider/physician's recommendation. For example, a woman said:

“Many people don't know about mammography, they don't know anything; even (they)'ve not heard the name of mammography." (Participant number 20)

Another woman said:

"It's not necessary, I went to the doctor, I'd been much examined by gynecologist but they've never suggested me to get mammogram. I think, they'll recommend it to me if it's needed." (Participant number 17)

\section{2) Misunderstandings}

Women believed that breast self-examination was sufficient to detect breast masses and lumps. In addition, they said that mammography would be required after detection of masses or lump during breast self-examination. Some women believed that the cause of breast cancer was due to unhealthy lifestyles such as eating in fast foods, ingesting chemical food additives, and lack of eating natural foods, stress, anxiety, depression, and melancholy. Therefore, in our participants' opinions, people who have a healthy lifestyle are not at risk of breast cancer and do not need to get a mammogram. For example, a participant said:

"I think breast self-examination, before getting mammogram, is very effective and mammogram is best to be done after detecting mass during breast self-examination." (Participant number 7)

“I think mammography is not needed, after that, when I don't have pain, I don't need mammography, and when someone feels pain in her breasts, mammogram is required." (Participant number 18).

\section{Fear Control}

Three subcategories were drawn from category fear control: (1) perceived fear, (2) denial of the risk of breast cancer, and (3) asymmetrical breast shape.

\section{1) Perceived fear}

Most of our participants expressed their fear of being diagnosed with breast cancer as a reason for not getting mammograms. In addition, they reported that just the imagining and thinking about the consequences of breast cancer such as chemotherapy, pain, death from 
cancer, suffering due to cancer, treatment costs, and mastectomy or having unattractive breasts made them more hesitant in getting mammograms. Based on this perspective, they would ultimately decide not to undergo mammography. Furthermore, some of the participants were worried about the harms of mammography with respect to squeezing/smashing the breast and radiation. In addition, many women perceived mammography as a painful procedure. For example, a woman said:

"Mammography was painful, it was terrible, I know women who did not feel pain during mammography but I was so annoyed." (Participant number 15)

Another woman also said:

"I'm afraid, because one of our close relatives was under chemotherapy, which makes me nervous. She was in front of my eyes, she was younger than me, and I can see her struggling every day." (Participant number 23)

\section{2) Denial of the risk of breast cancer}

Being afraid of facing the breast cancer diagnosis and self-denial were the reasons for many women's lack of going to mammography clinics. They believed that being unaware of breast cancer was better than being diagnosed. Some women said that they did not want to think about sickness and cancer; therefore, they did not undergo mammograms. A woman said:

"I feel that there's no disease in my body and I have a healthy body, so I don't think that I will get sick." (Participant number 18)

Another woman said:

"My main reason is my own positive view, I never think about sickness and illness, I always think about health." (Participant number 19)

\section{3) Asymmetrical breast shape}

Some of the participants expressed that women have unpleasant feelings regarding their breast size, and thus they do not wish to expose their breasts in public. This can result in the adversity toward undergoing mammography. A woman said:

"It's possible that somebody who has small, big, and badly-shaped breasts or has skin disease does not like to show her breasts to others." (Participant number 13)

\section{Prioritizing the Needs versus Mammography Preference}

Three subcategories were drawn from category priority of needs to mammography consisting of (1) family needs, (2) difficulties in referring to mammography centers, and (3) lack of income/economic bar- riers.

\section{1) Prioritizing needs}

The participants reported that they had other problems and issues that need to be prioritize first than mammography. They also said that they did not want to upset their family, especially their children, and therefore they avoided to disclose or pursue their health problems. For example, a woman said:

"I have a lot of problems, my parents and my daughter are sick, I have to take them to the doctor and take care of them, so I can't pay more money for mammography." (Participant number 12)

\section{2) Difficulties in referring to mammography centers}

The lack of convenient access to mammography centers due to commuting long distances and transportation difficulties were reported as some of the barriers to mammography adoption. Lack of time due to other responsibilities such as being married, taking care of children, and being employed were some other barriers that prevented the participants from undergoing mammography. For example, a woman said:

“Lack of time is one of my problems, I don't have enough time, I'm at work until noon, after returning home, I have to take care of my children and take them to the extra educational classes, and then I have to cook dinner." (Participant number 1)

\section{3) Lack of income/economic barrier}

Almost all participants pointed to having or not having health insurance and the high costs associated with mammograms. High treatment costs and lack of a comprehensive coverage by health insurance adversely affect the utilization of mammography screening. Cost was important particularly for women who did not have health insurance or had low incomes. For example, a woman said:

"For me, costs are more important than anything, I have much more urgent issues in my life [so] that I say to myself when I don't have pain why pay for mammogram." (Participant number 10)

\section{Inadequate Competency of Mammography Centers and Technicians}

This category consisted of two subcategories, (1) lack of mammography centers and technicians and (2) apathy of healthcare providers.

\section{1) Lack of mammography centers and technicians}

Most of the women complained of busy mammography centers, particularly public ones; they reported that the number of mammography centers and devices were not proportional to the population. They also reported of the time-consuming process of the entire screening, from making the appointment to receiving the test results. Distrust in the 
medical diagnosis and the physicians because of lack of expertise and necessary skills and also diagnostic errors represented as the most common complaints of the participants. For example, a woman said:

"The mammography process is troublesome, making the appointment, long waiting time at the office, getting the mammogram result on another day." (Participant number 5)

Another woman also reported:

"Because of the errors that we are seeing and hearing about medical examinations such as the errors in laboratory, scanning, and mammography, lack of skills of healthcare providers to work well with a mammography machine, their diagnosis is wrong." (Participant number 13)

\section{2) Clients-healthcare provider apathy}

Participants also believed that some of the health professionals in the mammography clinics had negative attitudes toward mammograms and sometimes suggested to the clients not to get mammography, making them dubious about getting mammograms. Some of the professionals dealt disrespectfully with the needs and concerns of the women and also did not have good communication skills. Lack of respect on the privacy of the referred women was also one of the barriers they identified. For example, one of the participants said:

"I went to the hospital and asked about mammography, healthcare providers told me that a lot of rays enter the body during mammography, and suggested that I should get an ultrasound first, and if the result was suspicious, then get mammogram." (Participant number 6)

“Mammogram techs don't treat me with respect by saying, you don't know how to put your breasts inside the machine. I was humiliated, it was the first time that I underwent mammography, she didn't explain to me how to prepare for mammography, I didn't know what to do." (Participant number 4)

\section{A sense of Losing Family Support}

This category consisted of two subcategories, i.e., (1) loss of family support and (2) disrupted body image stigma.

\section{1) Loss offamily support}

Lack of support, both personally and financially, played a role in mammography adherence by some women. They mentioned reasons, such as illiteracy and aging, due to which some women could not undergo mammography alone and needed assistance. Lack of support and cooperation of the husband was also reported as one of the barriers to mammography. Destruction of married life in case of detection of breast cancer was the issue that most women were concerned about. Lack of empowerment and unfamiliarity with one's own rights were also some of the other issues. In their opinions, these problems could affect women's health especially those who undergoing mammography. For example, a woman said:

“Some husbands don't allow their wives to do mammogram, I mean, in our region husbands do not care about their wives, if they find out that their wives are ill, they abandon them. Women aren't financially independent, they're dependent on their husbands (and). For this reason, they can't refer for mammography." (Participant number 8)

\section{2) Disrupted body image stigma}

Some participants believed that women felt that being physically fit and having well-shaped breasts is attractive image through the other's point of views,therefore mastectomy or other procedures such as chemotherapy might threaten this image. In addition, the taboo of breast cancer and being tagged as cancerous were reported to force women to evade mammography. They also believed that mammography was embarrassing and unpleasant. In addition, having a belief in fatalism and the inability to change fate were some of the barriers they identified. For example, one of the participants said:

"I say to myself, my fate be whatever it'll happen, for this reason I say why, by getting mammogram, I increase my problems and anxiety." (Participant number 13)

"For example, if a single woman faces some problems in her breasts, maybe her parents tell her why these changes are made, this word 'why' can be in the minds of anyone else in our country, maybe they would think that a series of provocations have led to these changes, for example, because of sexual contact or stimulation." (Participant number 15)

\section{DISCUSSION}

This qualitative study aimed to explore how Iranian women explain the barriers that they perceive as preventing them from undergoing mammography with special emphasis on cultural aspects. Ample evidence has indicated that individually perceived barriers are important predictors of healthy behaviors and behavioral change. ${ }^{13)}$

According to our findings, one of the perceived barriers to mammography was 'unawareness of mammography.' This factor highlights the influence of insufficient knowledge and lack of information on women's decisions regarding mammography. Other researchers have also reported lack of knowledge and misunderstandings regarding mammography, screening, and breast cancer as barriers to mammography screening. ${ }^{7,15,16)}$ In addition, evidence indicates that Iranian women are less aware of the screening programs in the healthcare system of their country and the vast majority of patients are diagnosed only in advanced stages. ${ }^{7,17)}$ This must cause policy makers to pay more attention on establishing suitable diagnostic and treatment facilities and improving breast cancer control policies. ${ }^{8,18)}$

Besides that, similar to our results, lack of symptoms and lack of a 
family history of breast cancer that therefore decrease the risk of developing breast cancer have been found to cause the women to think that they do not need to undergo mammography. ${ }^{7,19)}$ Moreover, most women believe that only if healthcare providers or physicians recommend getting a mammogram, they would comply willingly. This indicates that insufficient education and inadequate access to healthcare services represent an important perceived barrier to health-promoting behaviors. ${ }^{20)}$ It seems that access to regular screening mammography is highly dependent on recommendation and referral by a primary care provider, $^{21,22)}$ and physicians' recommendation for screening and counseling with female healthcare providers in healthcare centers may be influential in increasing mammography rates. ${ }^{23)}$

Healthcare providers in primary healthcare centers can also, through media, play an important role in promoting awareness of mammography and correcting false beliefs.

One of the identified themes in our study was fear control. Being afraid of various aspects of breast cancer can affect women's perceptions regarding the ways of responding to mammography results and relevant recommendations. Based on our results, imagining and thinking about negative consequences of breast cancer tend to affect women's decisions pertaining to undergoing mammography. Our participants' viewpoints regarding the role of fear were consistent with the results of a study conducted in the United States on a group of Jordanian and Palestinian immigrant women. Those women reported having certain fears such as fear of chemotherapy and body changes that take place after examinations and treatment, fear of pain, and fear of death. Thus, they preferred not to know rather than to know, because they believed that diagnosis of breast cancer was equal to end and death. ${ }^{14)}$ In addition, harmfulness of mammography was considered as another barrier on getting mammograms as the women expressed their concerns about potential side effects of the mammogram procedure itself; for example, X-ray exposure ${ }^{7,19)}$ and squeezing/ smashing of the breast during mammography may lead to breast cancer. $^{19)}$

Several behavioral change theories can explain the role of fear in the formation of behavior. Based on the Health Belief Model and Witte's Extended Parallel Process Model to assess the occurrence of permanent behavioral changes, one must feel threatened by his/her current behavioral patterns (perceived susceptibility and severity), i.e., women should first believe that they may develop a disease or condition and then perceive it as sufficiently life-threatening to take action for it. ${ }^{13,24)}$ In addition, based on the third construct of the transactional model of stress and coping process known as secondary appraisal, a person determines how much control he/she has over the stressors; then, based on this appraisal, he/she is engaged in either problem- or emotion-focused coping. Therefore, if individuals are not engaged in the problem-focused coping, they are likely to avoid stressful situations or deny the existence of a stressful situation and, instead, look on the brighter side of life. ${ }^{25)}$

Body image, obvious changes in the body shape after mastectomy, and unpleasant feelings about the size and shape of one's breasts, new roles as an 'unattractive' women, or interference in sexual relationships can have negative impacts on woman's decision to undergo mammography screening. ${ }^{26)}$

As a result, public health professionals and healthcare providers should provide coherent information about the benefits of mammography and the importance of early diagnosis of breast cancer. These may help women overcome their fear of mammography and to make informed decisions regarding getting them done.

Assuming various routine responsibilities and facing certain challenges may be considered important barriers to mammography adoption. This barrier is called conflicting priorities, ${ }^{23)}$ competing priorities or lack of giving priority to one's own health. ${ }^{14,27)}$ Women in one study reported that everything else comes first, and we forget about ourselves and taking care of ourselves. Generally, familial needs comprise the top priority in the lives of Iranian women. ${ }^{9)}$ In addition, consistent with our findings, high cost of living and medical services, ${ }^{7,27)}$ not having insurance, ${ }^{7,27)}$ inaccessibility, ${ }^{14,27)}$ transportation difficulties, ${ }^{14,27)}$ inconvenient facility location, ${ }^{7)}$ and distance ${ }^{23)}$ are some factors that interfere with undergoing mammography. Generally, the cost of healthcare services is a commonly reported barrier to health-related behaviors, particularly screening. These costs influence people's prioritization of the needs, especially in low-income countries.

Distrust in the medical diagnosis and the physicians because of potential lack of expertise and skills, as well as diagnostic errors, comprised one of the most common complaints of the study participants. Consistent with our findings, inability to get a doctor's appointment, ${ }^{19)}$ long period of waiting for confirmation of appointment, waiting a long time at the mammography centers, and waiting a long time to receive the test result were reported as the perceived barriers to mammography screening in other studies. ${ }^{7,19)}$

In some studies, the participants expressed doubts about the tests' accuracy and technicians' skills and therefore distrust in the healthcare system. ${ }^{24)}$ In addition, lack of the technicians and other healthcare providers' respect on the needs and concerns of women, lack of good communication skills, and lack of respect on the privacy of the referred women prevented them from undergoing mammography.

Addressing the patients' barriers and clearly and thoroughly explaining screening procedures have been significantly correlated with screening adherence. ${ }^{28)}$ Furthermore, lack of equipment, qualified professionals, and equipped facilities can adversely affect screening behaviors. ${ }^{14)}$

Lack of familial and social support might be influential in adhering to breast cancer screening. ${ }^{23)}$ Breast cancer has certain social implications such as divorce and, in some cases, feeling the loss of femininity. In some cultural subpopulations, husbands of women with cancer are advised by their families to abandon their wives and marry another woman to achieve sexually satisfying relationships, which is consistent with the observations in this study. Consistent with our results, men, in some cultures, have been reported to disapprove of undergoing breast cancer screening services and forbid women to refer to healthcare centers. ${ }^{14)}$ Believing in fatalism was drawn as another barrier to 
mammography. This is supported by a systematic review in which $64 \%$ of the studies showed a statistically significant association between fatalism and utilization of cancer screening services and the belief that all events that comprise the life of an individual are determined by God. Believing in fatalism can therefore serve as one of the barriers to undergoing mammography. ${ }^{20)}$ Some women in our study believed that having breast cancer was due to divine providence, ${ }^{14,19)}$ and if God has willed them to undergo mammography for breast cancer, they will ultimately do it; however, it was found that believing in God and prayer played an important role in preventing cancer. ${ }^{14)}$ It has been reported that women with breast cancer prefer no one to know about their disease and generally consider it a sign of shame, signifying that a woman with breast cancer is damaged and worthless, ${ }^{14)}$ which is consistent with our findings. As mentioned in the introduction, certain types of barriers might be more or less important for specific cultural or ethnic subpopulations, such as modesty among Asian-American women and embarrassment, cost of services among African American women, ${ }^{13)}$ and stigmatization among Arab women who are living in the United States. ${ }^{14)}$ Therefore, before the development and implementation of interventions to encourage women to adhere to mammography, relevant barriers throughout the mammography process that are important and dominant factors with respect to women's decision in the target population in refusing or accepting mammography screening should be identified. ${ }^{29)}$

\section{Limitations}

Limitations include small sample size, which is due to the design of the study, and purposive selection of the participants only from three cities of Iran, which limits the representativeness of the samples and generalizability of the results. Further, we did not triangulate the results of our study with quantitative approaches for this component.

\section{Conclusion}

In summary, this study showed different barriers that influenced women's decisions to undergo mammography, including insufficient information about mammography, psychological barriers, prioritizing needs over mammography, inadequate competency of mammography centers and technicians, and a sense of losing family support. These barriers were observed at various levels, i.e., individual, intrapersonal, social, healthcare system, and policy. Behavioral changes are expected to be maximized when environments and policies support health-promoting practices, when social norms and social support for health-promoting behaviors are strong, and when individuals are adequately motivated and educated to adopt such behaviors. Community-based awareness-promoting plans for women should be implemented with respect to sociocultural contexts. To undergo mammography for breast cancer detection at an early stage, all available barriers at different levels must be considered, and relevant interventions, adapted to each level, should be implemented. Providing an adequate number of affordable and conveniently accessible mammography centers, which entails employment of educated female healthcare workers, and creating support groups, may also play an important role in increasing Iranian women's participation in breast cancer screening. The collaboration between public health professionals, healthcare providers, and policymakers is needed to minimize the barriers women face in breast cancer screening.

\section{CONFLICT OF INTEREST}

No potential conflict of interest relevant to this article was reported.

\section{FUNDING}

This work was supported by the Tabriz University of Medical Sciences.

\section{ACKNOWLEDGMENTS}

The authors hereby express their gratitude to all women who participated in this study.

\section{ORCID}

Shayesteh Shirzadi: https://orcid.org/0000-0002-0914-4989

Hamid Allahverdipour: https://orcid.org/0000-0003-3700-6185

Manoj Sharma: https://orcid.org/0000-0001-5215-9740

Hadi Hasankhani: https://orcid.org/0000-0002-6710-5582

\section{REFERENCES}

1. Kashi AS, Yazdanfar S, Akbari ME, Rakhsha A. Triple negative breast cancer in Iranian women: clinical profile and survival study. Int J Cancer Manag 2017;10:e10471. https://doi.org/10.5812/ijcm.10471.

2. Davari M, Maracy MR, Emami MH, Taheri D, Aslani A, Givi M, et al. The direct medical costs of colorectal cancer in Iran; analyzing the patient's level data from a cancer specific hospital in Isfahan. Int J Prev Med 2012;3:887-92.

3. Zarei M, Bagheri-Saweh MI, Rasolabadi M, Vakili R, Seidi J, Kalhor $\mathrm{MM}$, et al. Breast cancer research in Iran: a scientometric analysis of publications output from 1991 to 2015 in Scopus. Electron Physician 2017;9:3816-22.

4. Enayatrad M, Amoori N, Salehiniya H. Epidemiology and trends in breast cancer mortality in iran. Iran J Public Health 2015;44:430-1.

5. Karbakhsh M. ABC of breast cancer awareness. Arch Breast Cancer 2014;1:77-8.

6. Corbex M, Burton R, Sancho-Garnier H. Breast cancer early detection methods for low and middle income countries, a review of the evidence. Breast 2012;21:428-34.

7. Watson-Johnson LC, DeGroff A, Steele CB, Revels M, Smith JL, Justen E, et al. Mammography adherence: a qualitative study. J Womens Health (Larchmt) 2011;20:1887-94.

8. Sarma EA. Barriers to screening mammography. Health Psychol Rev 2015;9:42-62.

9. Husain M, Orlowski MA, Wonders K, Hallam JS. Revisiting the relationship between beliefs and mammography utilization. Am J Health 
Stud 2010;25:78-85.

10. Allahverdipour H, Asghari-Jafarabadi M, Emami A. Breast cancer risk perception, benefits of and barriers to mammography adherence among a group of Iranian women. Women Health 2011;51:204-19.

11. Hardin AL. Fear as a barrier in mammography screenings. Kaleidoscope 2012;10:10.

12. Lee-Lin F, Menon U, Pett M, Nail L, Lee S, Mooney K. Breast cancer beliefs and mammography screening practices among Chinese American immigrants. J Obstet Gynecol Neonatal Nurs 2007;36:212-21.

13. Glanz K, Rimer BK, Viswanath K. Health behavior and health education: theory, research, and practice. Hoboken (NJ): John Wiley \& Sons; 2008.

14. Kawar LN. Barriers to breast cancer screening participation among Jordanian and Palestinian American women. Eur J Oncol Nurs 2013;17:88-94.

15. Corrarino JE. Barriers to mammography use for Black women. J Nurs Pract 2015;11:790-6.

16. Davis TC, Arnold CL, Rademaker A, Bailey SC, Platt DJ, Reynolds C, et al. Differences in barriers to mammography between rural and urban women. J Womens Health (Larchmt) 2012;21:748-55.

17. Harirchi I, Kolahdoozan S, Karbakhsh M, Chegini N, Mohseni SM, Montazeri A, et al. Twenty years of breast cancer in Iran: downstaging without a formal screening program. Ann Oncol 2011;22:93-7.

18. Asadzadeh Vostakolaei F, Broeders MJ, Mousavi SM, Kiemeney LA, Verbeek AL. The effect of demographic and lifestyle changes on the burden of breast cancer in Iranian women: a projection to 2030 . Breast 2013;22:277-81.

19. Kissal A, Beser A. Knowledge, facilitators and perceived barriers for early detection of breast cancer among elderly Turkish women. Asian Pac J Cancer Prev 2011;12:975-84.

20. Espinosa de Los Monteros K, Gallo LC. The relevance of fatalism in the study of Latinas' cancer screening behavior: a systematic review of the literature. Int J Behav Med 2011;18:310-8.

21. Keshinro A, Hatzaras I, Rifkind K, Dhage S, Joseph KA. The impact of primary care providers on patient screening mammography and initial presentation in an underserved clinical setting. Ann Surg Oncol 2017;24:692-7.

22. Plourde N, Brown HK, Vigod S, Cobigo V. Contextual factors associated with uptake of breast and cervical cancer screening: a systematic review of the literature. Women Health 2016;56:906-25.

23. Bowser D, Marqusee H, El Koussa M, Atun R. Health system barriers and enablers to early access to breast cancer screening, detection, and diagnosis: a global analysis applied to the MENA region. Public Health 2017;152:58-74.

24. Nadalin V, Maher J, Lessels C, Chiarelli A, Kreiger N. Breast screening knowledge and barriers among under/never screened women. Public Health 2016;133:63-6.

25. Shirzadi S, Nadrian H, Asghari Jafarabadi M, Allahverdipour H, Hassankhani H. Determinants of mammography adoption among Iranian women: what are the differences in the cognitive factors by the stages of test adoption? Health Care Women Int 2017;38:956-70.

26. Thomas E. From qualitative data to instrument development: the Women's Breast Conflict Scale. Qual Rep 2011;16:908-32.

27. Filippi MK, Pacheco J, James AS, Brown T, Ndikum-Moffor F, Choi WS, et al. American Indian men's perceptions of breast cancer screening for American Indian women. J Health Dispar Res Pract 2014;7:25-34.

28. Peterson EB, Ostroff JS, DuHamel KN, D’Agostino TA, Hernandez M, Canzona MR, et al. Impact of provider-patient communication on cancer screening adherence: a systematic review. Prev Med 2016;93: 96-105.

29. Lee H, Kim J, Han HR. Do cultural factors predict mammography behaviour among Korean immigrants in the USA? J Adv Nurs 2009;65: 2574-84. 